angina-related rehospitalization rate. The combined mortality or spontaneous $\mathrm{MI}$ rate was similar in the two groups, as were 4-year all-cause and cardiovascular mortality. The Kaplan-Meier curves for the two strategies followed similar patterns for each primary end point throughout follow-up. Revascularization rates were, note the authors, relatively high in the selective-invasive group, and optimum medical therapy was administered.

Original article Hirsch A et al. (2007) Long-term outcome after an early invasive versus selective invasive treatment strategy in patients with non-ST-elevation acute coronary syndrome and elevated cardiac troponin T (the ICTUS trial): a follow-up study. Lancet 369: 827-835

\section{Financial burden of care relates to worse outcome after AMI- even among the insured}

Health-care costs are increasing, and patients cannot always afford to access medical services. Rahimi and colleagues investigated whether monetary barriers were linked to compromised recovery from acute myocardial infarction (AMI).

When admitted to 1 of 19 US medical centers for AMI, $98 \%$ of the 2,498 patients in the PREMIER study responded to questions about avoiding treatment in the previous year because of cost. Outcomes were assessed 12 months later.

At baseline, financial barriers to accessing health-care services were reported by $18.1 \%$ of patients, of whom $68.9 \%$ had health insurance. During follow-up, people reporting financial restrictions were more likely to have angina ( $29.6 \%$ vs $17.5 \%)$, to have been hospitalized for any cause $(49.3 \%$ vs $38.1 \%)$, and to have a lower quality of life (Seattle Angina Questionnaire [SAQ] score 77.9 vs 86.2; $P<0.001$ for all) than those who had not reported monetary constraints. In addition, almost $13 \%$ of respondents failed to take prescribed medication as instructed in the year leading up to admission for AMI because of cost. More than $68 \%$ of these 316 patients were insured. Again, there were higher prevalences of angina (34.9\% vs $17.9 \%)$ and allcause rehospitalization (57.0\% vs $37.8 \%)$, and a poorer quality of life (SAQ score 74.0 vs $86.1 ; P<0.001$ for all) in those reporting financial barriers.
The discrepancies reported above persisted after adjustment for potential confounding factors such as insurance and socioeconomic status, indicating that underinsurance-not just lack of insurance-can hinder post-AMI recuperation.

Original article Rahimi AR et al. (2007) Financial barriers to health care and outcomes after acute myocardial infarction. JAMA 297: 1063-1072

\section{Multiple disease locations increase risk of cardiovascular events in atherothrombosis}

To date, the majority of research into atherothrombosis has been carried out in single geographical regions, or has focused on particular vascular beds. Steg et al. have analyzed data from the global REACH registry $(68,236$ outpatients; 44 countries), and have reported cardiovascular-event rates for patients with documented coronary artery disease (CAD), cerebrovascular disease (CVD) or peripheral arterial disease (PAD).

In total, 1-year outcomes were available for just over $95 \%$ of patients. Overall mortality was $2.58 \%$ and cardiovascular-related mortality was $1.65 \%$. The incidence of a composite of cardiovascular death, myocardial infarction or stroke was $4.24 \%$. This end point was observed in $4.52 \%, 6.47 \%$, and $5.35 \%$ of patients with documented CAD, CVD or PAD, respectively, but in only $2.15 \%$ of patients with risk factors for atherothrombosis but no documented disease. For patients with established CAD, CVD or PAD, the incidences of a major cardiovascular event or hospitalization for atherothrombotic events were $15.20 \%, 14.53 \%$, and $21.4 \%$, respectively. These rates increased with the number of arterial disease locations, ranging from $5.31 \%$ for patients with atherothrombotic risk factors only, to $26.27 \%$ for patients with CAD, CVD and PAD $(P<0.001$ for trend).

The authors anticipated a 3.0\% event rate for the composite end point of cardiovascular death, myocardial infarction or stroke; interestingly, this estimate was exceeded in all geographical regions. Evaluation of the causes of the low cardiovascular-event rates observed in regions such as Japan might provide important clinical insights, suggest the authors. They conclude that physicians should be aware of 